\title{
ESTUDOS SOBRE A ALIMENTAÇÃO MINERAL DO ALGODOEIRO. V. Efeito das Carências Combinadas no Crescimento, Aspecto e Composição Química *
}

\author{
F. Freitez Perez ** \\ E. Malavolta ***
}

\section{RESUMO}

Foi estudado o efeito das carências combinadas de alguns macronutrientes no crescimento, aspecto e composição mineral do algodoeiro, Gossypium hirsutum L., var. IAC RMB.

O trabalho regstra: sintomas foliares dos macronutrientes estudados nas folhas superiores e inferiores das plantas.

\section{INTRODUÇÃO}

A literatura sobre a nutrição mineral do algodoeiro fornece informações sobre o efeito das deficiências individuais de macronutrientes no aspecto, crescimento, produção, composição química do algodoeiro (veja-se por exemplo a revisão de MALAVOLTA \& HAAG, 1968). Não se encontram, porém, observação e dados a respeito das alterações que se dão quando mais de um elemento está em falta ao mesmo tempo, o que justifica este trabalho, uma vez que tal situação poderá se verificar em condições de campo.

\section{MATERIAL E MÉTODOS}

1. O algodoeiro foi cultivado em solução nutritiva de HOAGLAND E ARNON (1950) de modo a fornecer os seguintes tratamentos, com 4 repetições:

* Parte da tese apresentada pelo primeiro autor em cumprimento parcial das exigências para a obtenção do título de "Magister Scientiae".

** Centro Exptal. de Estudos Superiores, Barqto., Lara, Venezuela.

*** Depart. Química - ESALQ - Piracicaba. 


\begin{tabular}{|c|c|}
\hline tratamento & solução \\
\hline $1 .^{\circ}$ & completo \\
\hline $2 .^{\circ}$ & $-\mathrm{N}$ \\
\hline $3 .^{\circ}$ & $-P$ \\
\hline $4 .^{\circ}$ & $-\mathrm{K}$ \\
\hline $5 .^{\circ}$ & $-\mathrm{Ca}$ \\
\hline $6 .^{\circ}$ & $-\mathrm{Mg}$ \\
\hline $7 .^{\circ}$ & $-s$ \\
\hline $8 .^{\circ}$ & - NP \\
\hline $9 .^{\circ}$ & - NK \\
\hline $10 .^{\circ}$ & - $\mathrm{NCa}$ \\
\hline $11 .^{\circ}$ & - NMg \\
\hline $12 .^{\circ}$ & - NS \\
\hline $13 .^{\circ}$ & - PK \\
\hline $14 .^{\circ}$ & - $\mathrm{PCa}$ \\
\hline $15 .^{\circ}$ & - $\mathrm{PMg}$ \\
\hline $16^{\circ}$ & - PS \\
\hline $17 .^{\circ}$ & $-\mathrm{KCa}$ \\
\hline $18 .^{\circ}$ & $-\mathrm{KMg}$ \\
\hline $19 .^{\circ}$ & $-\mathrm{KS}$ \\
\hline
\end{tabular}

A colheita das plantas se fez quando os sintomas se mostraram evidentes, fazendo-se a separação das mesmas em: flores e frutos, folhas superiores (da metade da altura para cima), folhas inferiores, caule superiores e inferiores, galhos superiores e inferiores, raízes. Tomou-se o peso do material fresco e do material seco.

2. A análise química das folhas foi feito por métodos convencionais, o mesmo se dando com a análise estatística dos resultados.

\section{RESULTADOS E DISCUSSÃO}

1. Os dados de crescimento e produção deixam de ser registrados porquanto o critério fixado para o final do ensaio torna irrelevantes as comparações. Em todos os tratamentos - Ca e no - NMg houve queda das folhas mais velhas. 
2. Os sintomas produzidos pelas deficiências individuais de macronutrientes concordam em linhas gerais com os encontrados na literatura. Quando dois elementos foram omitidos de uma vez as seguintes possibilidades se verificaram:

foi mantida a individualidade;

o sintoma das carências de um elemento predominou sobre o do outro;

o sintoma é diferente do correspondente à falta individual;

o primeiro caso se manifestou geralmente quando as alterações foliares de um elemento se apresentaram nas folhas mais novas enquanto as do outro o fazem nas mais velhas, refletindo maior ou menor translocação ou redestribuição interna; o segundo caso se deu quando não há simultaneidade no aparecimento dos sintomas; o último caso, finalmente foi observado somente quando os elementos implicados estão muito relacionados na sua absorção, transporte e funções. Em resumo constatou-se o seguinte quadro geral significativo:

(a) - NP : sintomas ou carências de $\mathbf{N}$ nas folhas superiores e de $\mathbf{P}$ nas inferiores;

(b) - $\mathrm{NK}$ sintomas de carências de $\mathrm{N}$ nas folhas superiores e de $\mathrm{K}$ nas inferiores;

(c) - $\mathrm{NCa},-\mathrm{PCa}$ e $-\mathrm{KCa}$ : sintomas de carências de $\mathrm{Ca}$;

(d) $-\mathrm{MNg}$ : sintomas semelhantes aos causados pela falta individual;

(e) -NS : sintomas semelhantes aos causados pela falta de $\mathrm{N}$;

(f) - PK: sintomas semelhantes aos causados pela falta de P;

(g) - PMg: perda de turgidez, secamento e queda das folhas;

(h) -PS : manchas pardacentas no limbo, espessamento dos bordos;

(i) - KMg: sintomas semelhantes aos das carências individuais;

(j) - KS: sintomas de carências de $\mathrm{K}$ nas folhas inferiores e do $S$ nas superiores.

3. Os teores encontrados nas folhas superiores (F.S.) e nas folhas inferiores, (F.I.) aparecem no Quadro 1. O exame dos dados mostra, entre outras coisas que:

(a) quando $\mathrm{N}, \mathrm{K}$ e $\mathrm{S}$ foram omitidos um de cada vez caiu a $\%$ do elemento nas folhas superiores; 


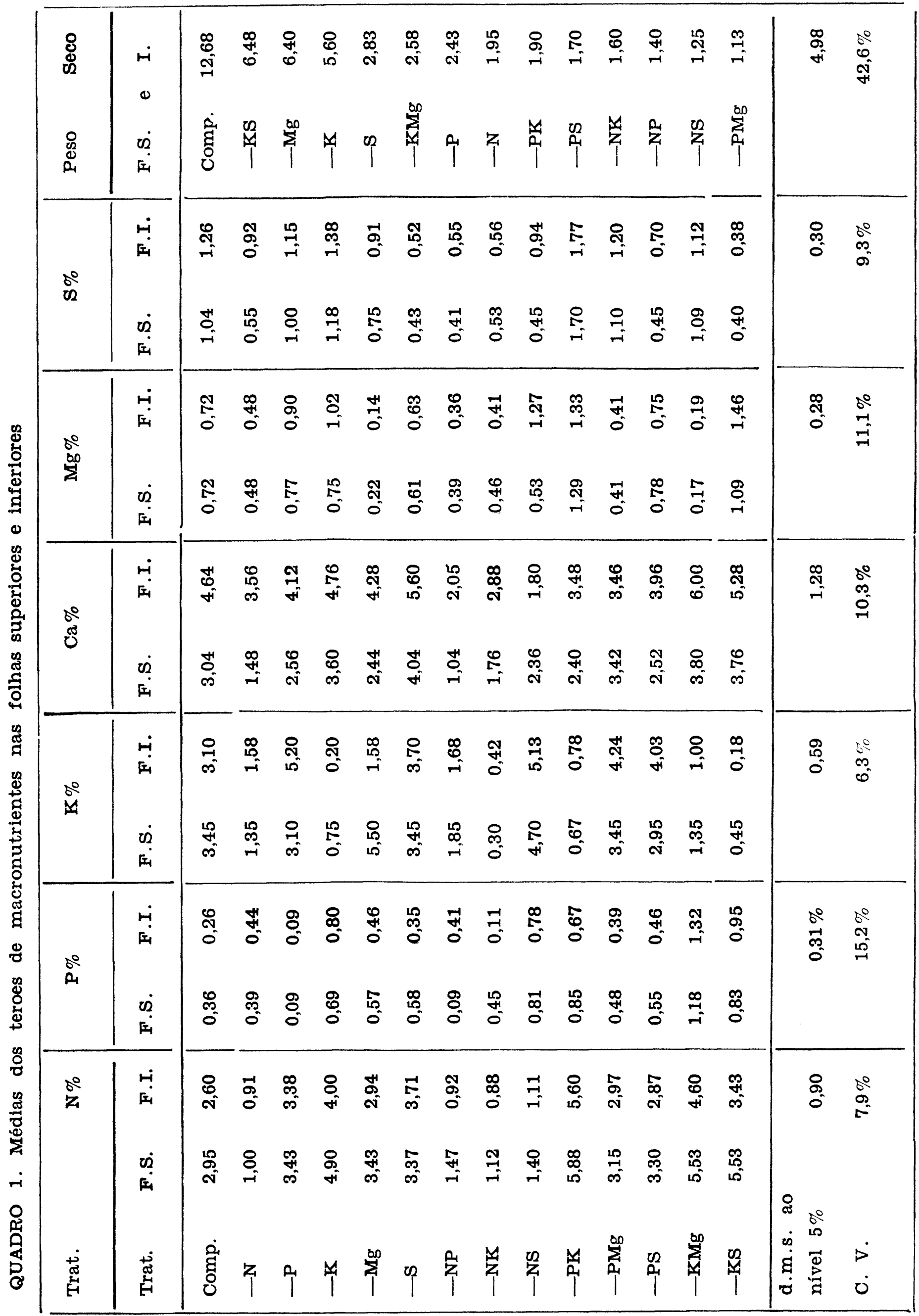


(b) a omissão individual de $\mathrm{N}, \mathrm{K}, \mathrm{Mg}$ e $\mathrm{S}$ causou diminuição no teor correspondente das folhas inferiores;

(c) diversas interações se verificaram como consequência das carências simples e combinadas (efeitos na absorção e translocação e da diluição).

\section{CONCLUSÕES}

Sementes de algodoeiro (Gossypium hirsutum L., var. - IAC RM3) foram postas a germinar e as plantas assim obtidas foram transplantadas para tanques de folha contendo 18 litros de solução nutritiva, modificada, de HOAGLAND \& ARNON (1950) diluída a um quinto $(1 / 5)$.

Sete dias após o transplante, foram transferidas as mudas para solução nutritiva, modificada, de HOAGLAND \& ARNON (1950) diluída à metade.

Depois de sete dias foram colocadas nos diferentes tratamentos, cada um com 4 plantas.

Os tratamentos, em número de 19 , foram os seguintes:

$\begin{array}{cc}\text { Tratamento } & \text { Solução nutritiva } \\ 1 .^{\mathrm{a}} & \text { Completa } \\ 2 .^{\mathrm{a}} & \text { Sem N } \\ 3 .^{\mathrm{a}} & \text { Sem P } \\ 4 .^{\mathrm{a}} & \text { Sem K } \\ 5 .^{\mathrm{a}} & \text { Sem Ca } \\ 6 \mathrm{a}^{\mathrm{a}} & \text { Sem Mg } \\ 7 .^{\mathrm{a}} & \text { Sem S } \\ 8 .^{\mathrm{a}} & \text { Sem NP } \\ 9 .^{\mathrm{a}} & \text { Sem NK } \\ 10 .^{\mathrm{a}} & \text { Sem NCa } \\ 11 .^{\mathrm{a}} & \text { Sem NMg } \\ 12 .^{\mathrm{a}} & \text { Sem NS } \\ 13 .^{\mathrm{a}} & \text { Sem PK } \\ 14 .^{\mathrm{a}} & \text { Sem PCa } \\ 15 .^{\mathrm{a}} & \text { Sem PMg } \\ 16 .^{\mathrm{a}} & \text { Sem PS } \\ 17 .^{\mathrm{a}} & \text { Sem KCa } \\ 18 .^{\mathrm{a}} & \text { Sem KMg } \\ 19 .^{\mathrm{a}} & \text { Sem KS }\end{array}$


Os sintomas de deficiências de macronutrientes foram descritos pouco antes da colheita.

As plantas foram colhidas e divididas em: flores e frutos, folhas superiores (da metade dos nós para cima), folhas inferiores (da metade dos nós para baixo), caule superior, caule inferior, galhos superiores, galhos inferiores e raízes.

Analisaram-se as folhas superiores e infleriores por métodos químicos apropriados.

Constatou-se que as soluções carentes dos elementos $\mathrm{N}, \mathrm{K}$ e $\mathrm{S}$, um por vez, provocaram nas folhas superiores, uma queda da porcentagem do referido elemento, e a omissão dos elementos $\mathrm{N}, \mathrm{K}$, $\mathrm{Mg}$ e S, um por vez causaram nas folhas inferiores uma diminuição do teor do referido elemento.

Verificaram-se diversas interações entre os elementos, como consequência dos trataemntos simples e combinados.

Os tratamentos carentes de $\mathrm{Ca}$, simples ou combinados, e o tratamento - NMg, atuaram sobre as folhas inferiores produzindo uma defoliação total, permanecendo só as folhas superiores.

Os conteúdos porcentuais das folhas, referidos aos tratamentos Completos e $-\mathrm{N},-\mathrm{P},-\mathrm{K},-\mathrm{Ca},-\mathrm{Mg}$ e $-\mathrm{S}$, foram os seguintes :

$\begin{array}{cccc}\text { Elemento } & \text { Folhas } & \text { Deficiente } & \text { Adequado } \\ \text { N \% } & \text { superiores } & 1,00 & 2,95 \\ & \text { inferiores } & 0,91 & 2,60 \\ \text { P } \% & \text { superiores } & 0,09 & 0,36 \\ & \text { inferiores } & 0,09 & 0,26 \\ \text { K } \% & \text { superiores } & 0,75 & 3,45 \\ & \text { inferiores } & 0,20 & 3,10 \\ \text { Ca } \% & \text { superiores } & 2,00 & 3,04 \\ & \text { inferiores } & - & 4,64 \\ \text { Mg\% } & \text { superiores } & 0,22 & 0,72 \\ & \text { inferiores } & 0,14 & 0,72 \\ \text { S } \% & \text { superiores } & 0,43 & 1,04 \\ & \text { inferiores } & 0,52 & 1,26\end{array}$

\section{SUMMARY}

STUDIES ON THE MINERAL NUTRITION OF THE COTTON PLANT. V. EFFECT OF JOINT DEFICIENCIES ON GROWTH, ASPECT AND CHEMICAL COMPOSITION.

Symptoms of deficiencies of several macronutrients and binary combinations thereof were induced in the cotton plant. 
It was found that depending upon the particular combination the following picture developed:

(a) individual symptoms are present according to the general pattern;

(b) individual symptoms are present with a reversal of the general pattern with respect of distribution;

(c) a combination of the individual symptoms shows up;

(d) an individual symptom masks the other;

(e) a different symptom develops.

Chemical analysis of both upper and lower leaves has shown single and multiple effects depending upon the treatment considered.

\section{LITERATURA CITADA}

HOAGLAND, D. R. \& D. I., ARNON. 1950 - The water culture method for growing plants without soil. Calif. Agr. Exp. Sta. Cir. 341.

MALAVOLTA E. \& H. P. HAAG. 1968 - Recent advances on the study of the mineral nutrition of cotton in Brazil. An. E.S.A. "Luiz de Queiroz" XXV: 219-230. 
